\title{
An empirical approach to market partitioning: Application to the cigarette market
}

Received (in revised form): 27th February, 2004

\section{John Carter}

is Professor of Management Science at the Lubin School of Business, Pace University in New York. He teaches statistics, decision modelling, operations and quality management in the MBA programme. His research and consulting interests include market structure analysis, healthcare quality and operations analysis.

\section{Fred Silverman}

is Professor of Management Science at the Lubin School of Business, Pace University in New York. He teaches operations, quality, supply chain management, decision modelling and statistics in the MBA programme. His research and consulting interests are in market structure analysis, production systems design and healthcare quality.

\begin{abstract}
The market partitioning problem is to determine a hierarchy of attributes that define directly competing subsets of products. This paper describes an entropy-based methodology for unfolding the attribute partitioning structure of a market from diary panel purchase data. The methodology may be applied to any collection of attributes, including image attributes, of interest to the marketing manager. In addition, it can identify differences in attribute structures among key market segments. The paper reports on an application of the methodology to the cigarette market, concluding that the overall market structure includes both objective and image attributes at the top levels of the partitioning structure.
\end{abstract}

\section{THE PROBLEM}

An important brand management problem is to determine which product attributes are significant for explaining competition in the marketplace. If the manager understands that the firm's products compete strongly on some attributes (but not others), these attributes can be emphasised in designing, positioning, pricing and promoting products. Market partitioning seeks to identify those attributes that explain the

Professor Fred Silverman Lubin School of Business, Pace University,

1 Martine Avenue, White Plains, NY 10606, USA.

Tel: +1 914422 4184; Fax: +1 914422 4184; e-mail: fsilverman@pace.edu grouping of members of a product class into directly competing subsets. With each product alternative represented as a vector of attributes, the market may be modelled as a hierarchy of attributes, with top-level attributes imposing the most constraint on consumers' switching behaviour.

Methods applied to this problem so far have required hypothesising a hierarchy, then comparing observed switching with what might be expected under assumptions of market equilibrium. The method of this paper provides an evolutionary algorithm that automatically unfolds the hierarchy. It is a method that allows a large number of attributes to be included in the analysis — including physical and image - and unfolded without the need for prior judgments of relative importance in the market. This empirical approach identifies the partitioning structure of a market from switching matrices derived from diary 
panel data, using an entropy-based methodology. The methodology is described using a simple numerical example, and then applied to an analysis of the cigarette market. Finally, the implications of a market's attribute structure for decisions in pricing, promotion and new product design are discussed.

\section{OTHER APPROACHES TO THE PARTITIONING PROBLEM}

The Hendry Corporation first developed the attribute partitioning model of market structure as part of HendroDynamics, a system of models designed to determine optimal pricing and advertising levels. ${ }^{1,2}$ Kalwani and Morrison show that the underlying Hendry principle is that each partition consists of a set of heterogeneous multinomial consumers in which each brand in a partition has the same switching constant and this switching constant also applies to the partition as a whole. ${ }^{3}$ The Hendry approach begins with a hypothetical market structure based on expert judgment. Switching patterns are then computed within and across partitions and switching constants compared with those constants expected if the structure were correct. If observed switching is not consistent with the hypothesised structure, another structure is proposed. The process stops when there is a satisfactory fit. Rubinson et al. point out that the theoretical constants are intended to apply under equilibrium market conditions. ${ }^{4}$ Therefore there is some ambiguity as to whether a lack of concurrence between actual and expected switching constraints is due to an incorrectly hypothesised structure or a departure from equilibrium. In addition to the Hendry approach, several authors have proposed confirmatory analyses of $a$ priori hypothesised structures. ${ }^{5-7}$
Another approach to producing a Hendry-like 'hierarchical choice process' was developed by Rao and Sabavala who used hierarchical cluster analysis applied to switching matrices derived from diary panel data. ${ }^{8}$ Like the methodology described in the present paper, the process is exploratory. It begins with a list of brands in a market, clusters the brands based on a proximity measure computed from a brand switching matrix and forms a dendrogram, which is the inverse of the resulting hierarchy. Rao and Sabavala stress the need to choose a proximity measure that has intuitive appeal. This choice affects the results. After automatically developing the brand clusters, they use judgment to infer the attributes that explain the grouping. They say that the resulting hierarchy may be a spurious result of the hierarchical clustering. The partitioning method described in the present paper also uses an automatic algorithm, begins at the top of the tree and the hierarchy unfolds without the need for exercise of judgment. Furthermore, it explicitly treats product variants as vectors of their (known) attributes and brand name itself is one of the attributes. So unlike Rao and Sabavala's concept, brand name becomes a partitioning variable itself, residing anywhere from the top to the bottom of the attribute hierarchy.

The main differences between the partitioning approach described in this paper and other multivariate methods applied to attribute analysis derive from the purpose of the analysis, the data utilised and the modelling approach. The purpose of partitioning analysis is to identify groups of directly competing product variants, defined as attribute vectors. By contrast, market segmentation seeks to identify groups of consumers with similar purchase or usage behaviour or reactions to marketing efforts. ${ }^{9}$ The many multivariate methods that have 
been used to identify market segments include canonical analysis, factor analysis, cluster analysis, multidimensional scaling (MDS), segmentation trees and correspondence analysis.

The partitioning approach described here is based on different data from techniques used for perceptual mapping. Product attributes in partitioning analysis are treated as nominally-scaled variables. Thus methods such as factor analysis, which depend on correlations among metric variables, are not appropriate. A fundamental tenet of partitioning analysis is that the extent to which two products compete in a marketplace can be measured most realistically by observing actual consumer purchase behaviour in a sequence of purchase occasions. If a consumer is observed to purchase a subset of products to satisfy a particular need, those products are revealed as substitutes and therefore as closely competing with one another. By contrast, data used to develop spatial maps representing the positions of a set of brands in two or more dimensions are typically obtained in a laboratory setting or by a survey of selected respondents. The result is either a square matrix of judged similarities or preferences (used in MDS) or a rectangular matrix of frequencies with which each brand is associated with each attribute (used in correspondence analysis). Besides the fundamental difference between data used for partitioning versus perceptual mapping, the methods themselves have a different purpose. MDS seeks to produce a map in two or more dimensions based on distances computed from the data. The derived dimensions may well be consistent with particular product attributes or combinations thereof, yet are often difficult to interpret. In three or more dimensions, the interpretation of results is even more difficult. By contrast, the entropy-based methodology utilises the known attributes that users of the product might actually evaluate when choosing among product alternatives and judges importance of candidate attributes by revealed choices. Furthermore, the method allows the researcher to include image attributes such as 'outdoors' in addition to objective attributes like filter type in the cigarette context. The maps produced by correspondence analysis show attributes and the objects (brands or products) in derived space, but it is well known that results are sensitive to outliers and omitted attributes or objects.

The partitioning structure in this paper is tree-like. Other methods that provide tree-like structures are segmentation trees and means-end analysis. Winter proposed segmentation trees to map the decision process of customers, with a view to identifying segments of customers or potential customers who may be profitably targeted in different ways. ${ }^{10}$ As described by Bock and Styles, the approach requires the analyst to assemble a list of variables measuring relevant customer characteristics, rank the variables, using judgment, and detail the branches of the decision tree. ${ }^{11}$

Segmentation trees are not designed to deduce competitive product relationships from behavioural data, but rather to identify the variables that usefully group customers into homogeneous groups. Means-end analysis is based on intensive interviews with individual consumers to deduce linkages between product attributes and the consequences and values they affect. The structure is typically shown as a laddermap, which is tree-like with attributes at one level.

\section{ANALYTICAL APPROACH}

The starting point for identifying the hierarchical structure of attributes is to define all product alternatives as a vector of attributes, using all relevant objective 
Table 1: Product descriptions

\begin{tabular}{llll}
\hline Variant & Brand & Colour & Size \\
\hline A & MM & Red & S \\
B & TR & Orange & M \\
C & JJ & Red & L \\
D & JJ & Red & S \\
E & MM & Orange & XL \\
\hline
\end{tabular}

and subjective attribute dimensions. For example, tobacco products could be defined by the attributes: brand, filter type, package, lifestyle image, etc. One product might be the vector (Marlboro, filter, box, vigorous). In this paper the methodology for determining the market partitioning structure is based on the concept of entropy in consumer choice patterns. Even in markets characterised by a low level of switching, like the cigarette market, entropy measures provide a sensitive means of identifying structural properties of the market. The analysis is predicated on the premise that if switching behaviour is unrelated to anything other than the individual brand/styles themselves, the entropy of purchase behaviour will be the same for all attribute dimensions. Should this not turn out to be true and significant entropy differences are found among attributes, the existence of a hierarchy is indicated. Attribute dimensions exhibiting decreasing degrees of customer loyalty determine the attribute hierarchy. The attribute dimension exhibiting the greatest degree of consumer loyalty occupies the top level of the hierarchy. Product variants in top-level partitions are most closely competing, in the sense that there is strong resistance to switching from one partition to another. For tobacco, if filter type is at the top of the hierarchy, the Marlboro product described above would primarily compete with other filter products. Consumers would more readily substitute different brands, lengths and package types than choose a product without a filter. The second level in the hierarchy is the attribute dimension that exhibits the next highest loyalty within the primary partitions. This analysis continues for lower-level partitions.

In order to demonstrate how this model can be applied, an example of the fruit juice market is used. Consumers can select from among three brands, two colours (flavours) and four sizes. Not all choices are available for each brand. The three brands are Minute Maid (MM), Tropicana (TR) and Juicy Juice (JJ). The drinks come in red, cherry and orange-coloured flavours. Four sizes are available: small (S), medium (M), large (L) and extra large (XL). It is further assumed that only the five product variants shown in Table 1 (of a possible 24) are available.

An attribute hierarchy with the order size, colour, brand partitions the market into four competing subsets by size. Consumers in that market switch relatively little among sizes. Because of the particular colours available within sizes, there is no opportunity for consumers to switch among different colours unless they are prepared to change to a different package size. At the lowest level in the hierarchy, consumers have the least resistance to switching. If one brand is not available, another will do almost as well or as well. The attribute hierarchy may be shown as a graph (Figure 1). 


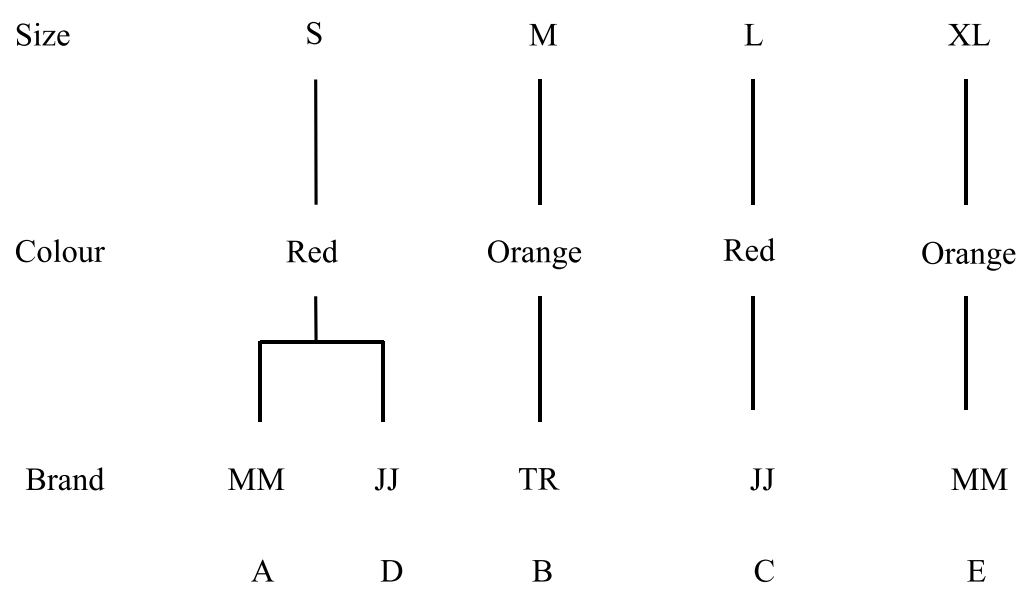

Figure 1 The size-colour-brand hierarchy

\section{SOURCES OF DATA}

In order to identify empirically how a set of product variants is partitioned, data reflecting the relationship among the variants are needed. A common way of trying to measure perceived relationships among attributes is to ask a structured set of questions of a sample of consumers. Aside from the high cost of obtaining such data for a large sample of individuals, one weakness of this approach is that respondents are placed in a hypothetical situation and reported relationship data may well be at odds with relationships revealed by actual purchase behaviour. The extent to which attributes are substitutable is more reliably measured by recording the attributes actually chosen to meet a particular need. For example, if a person were observed to eat at a coffee shop, then a fast-food restaurant, followed by a steak house, it would be fallacious to conclude that the three types of restaurant are closely competitive or close substitutes. Ideally then, context should be controlled for when collecting data on substitutability patterns. The methodology used in this paper is relevant for frequently purchased consumer products. It is not suitable for expensive, durable goods since the purchaser's utility function probably changes from one purchase occasion to the next. Diary panel data, though imperfect, are readily obtainable and usually record use context.

\section{ENTROPY IN MARKETING MODELLING}

The concept of entropy offers a natural means of measuring information. In the physical sciences, entropy has been viewed as a measure of the disorder, uncertainty or randomness in a probabilistic system. Brockett et al. state that 'the amount of uncertainty and the amount of information are inversely related; e.g., the information in an experiment can be regarded as the amount of uncertainty which would be eliminated by performing the experiment'. ${ }^{12}$ In a brand choice context, assume that a consumer buys brand $i$ with probability $p_{i}$. Entropy measures the amount of uncertainty in the choice process. For a consumer who is completely indifferent among all brands, the probabilities are all equal and entropy is at its highest. This indicates the maximum uncertainty associated with the 
choice. On the other hand, if a consumer is completely loyal to one brand, the probability of choosing that brand is one and zero for all others, giving an entropy or uncertainty of zero.

Shannon's definition of the entropy, $\mathrm{H}\left\{p_{i}\right\}$, of a stochastic system with $n$ states, having probability $p_{i}$ is given by:

$$
H\left\{p_{i}\right\}=-k \sum_{i=1}^{n} p_{i} \log p_{i}
$$

where $k$ is an arbitrary constant and the base of logarithms is arbitrary. ${ }^{13}$ Choosing $k=1$, the function has its maximum value of $\log n$ when the $n$ states are equally likely. Entropy theory holds that all systems will be at the maximum entropy consistent with the constraints on the system. Herniter uses this principle to develop a model of brand switching behaviour in an equilibrium market based only on market shares. ${ }^{14}$ In a later paper he compares this model with the Hendry model of consumer behaviour, which also relies on the equilibrium assumption. ${ }^{15}$ Information theory, which defines information conveyed about one random variable by another as a reduction of entropy, has been shown to be equivalent to traditional analysis of variance methods. Only information theory is appropriate when the criterion variable is measured on a nominal or ordinal scale. ${ }^{16}$ This work was recently extended to demonstrate that information theory is a unifying approach to a wide range of statistical methods. ${ }^{17}$ The capacity of entropy-based measures to deal with all levels of data contributes to their appeal as contending measures of attribute importance for partitioning analysis.

One of the strengths of the entropy measure is that it can be used to study dependencies in sequences of outcomes. First, second and third order dependencies may be analysed quite readily. In the present context, a first order process is assumed, where the last item purchased depends only on the one purchased the time before last. By dividing switching frequencies in a row by the total row frequency, rowconditional relative frequencies may be computed. These will be treated as conditional probabilities. From these conditional probabilities and market share probabilities, a measure of switching entropy is computed as:

$$
H_{s}=\sum_{i} p_{i} \sum_{j} p_{j \mid i} \log p_{j \mid i}
$$

where $p_{i}=$ attribute state $i$ 's share of the market and $p_{j \mid i}=$ the conditional probability of choosing $j$ next time given that $i$ was chosen last time. $H_{s}$ depends on the number of attribute states $(n)$ and has a maximum value $\left(H_{\max }\right)$ of $\log n$. This occurs when all conditional switching probabilities $\left(p_{j \mid}\right)$ are equal. ${ }^{18}$ In order to compare switching entropies for attributes with differing numbers of states, $H_{s}$ is normalised by dividing by $H_{\max }$, ie adjusted $H_{s}=H_{s} / \log n$.

\section{EMPIRICAL PARTITIONING METHODOLOGY}

The assumption underlying the attribute hierarchy is that consumers view product variants in a competitive subgroup as relatively close substitutes. A high-level attribute exhibits relatively low switching — and therefore switching entropy - across states (for example, from one size to another). Similarly, low-level attributes exhibit relatively high switching entropy.

\section{Algorithm}

1 Compute attribute switching matrices.

2 Compute the adjusted switching 


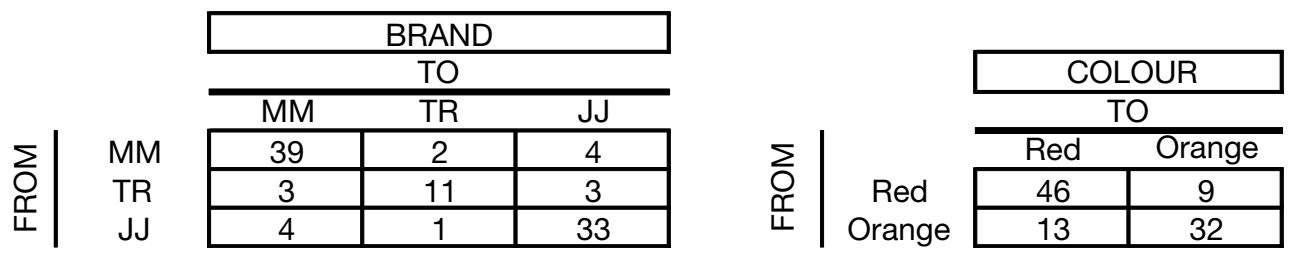

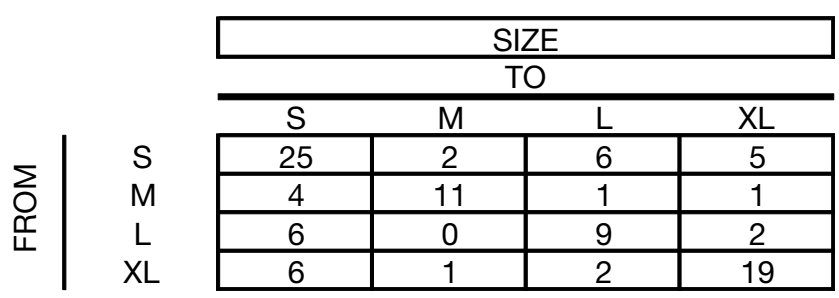

Figure 2 Attribute switching matrices

entropy for each attribute dimension.

3 Select the attribute dimension with the lowest entropy as the highest-level attribute in the hierarchy.

4 At the next level, only transaction pairs with higher-level attributes in common are considered in computing switching matrices for the remaining dimensions.

5 A weighted average switching entropy is computed using as weights the market shares of the attribute combinations at the level selected in step 4.

6 Select the attribute dimension with the lowest entropy as the highest-level attribute in the hierarchy.

7 Repeat steps 4 to 6 until all attribute dimensions have been selected.

\section{Applying the methodology}

In order to demonstrate how the algorithm works, the fruit juice market described earlier, in which consumers choose among five product variants, defined in terms of brand, colour and size attributes, is considered again.

\section{Level 1}

The first step is to compute attribute switching matrices, which are as shown in Figure 2.

The next step is to compute the switching entropy for each attribute dimension. The switching entropy value for Brand is 0.490 , for Size it is 0.688 and for Colour it is 0.744 . For example, the calculations for Colour are as shown in Figure 3.

At the third step, the attribute dimension with the lowest entropy is selected as the highest-level attribute in the hierarchy. In the example, Brand is at the top level.

\section{Level 2}

Since this is a brand primary market, only transaction pairs with common brand names are considered in computing brand-conditional switching matrices for Colour and Size within each brand.

A weighted average switching entropy is computed using as weights the market 
Example: Colour

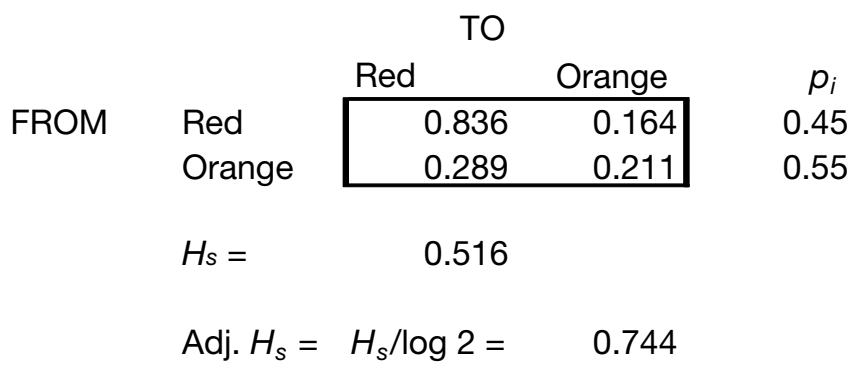

Figure 3 Entropy calculation example

shares of the brands. The adjusted conditional switching entropy values for the two second-level candidates is 0.363 for Colour and 0.712 for Size, thus indicating a Brand-Colour-Size hierarchy.

This attribute hierarchy indicates that consumers show relatively little loyalty to size variants, so that $\mathrm{A}$ and $\mathrm{E}$ are strongly competitive, as are D and C. Consumers are less likely to switch to $\mathrm{B}$ from the other variants, so $\mathrm{B}$ is less competitive and more immune to marketing efforts.

\section{APPLICATION TO THE CIGARETTE MARKET}

Many tobacco industry marketing executives believe that image qualities and objective product attributes play an important part in the development of the position of brands in the marketplace. Advertising themes amply demonstrate this belief when they project images such as vigorous lifestyle and masculinity as well as objective attributes like menthol content and length. Determining which product attributes (image and objective) are actually important for explaining competition in the marketplace has been difficult. The task is made difficult by the very large number of brands/styles in the cigarette market and the low levels of switching exhibited by smokers.
Conversations with inventory marketing analysts suggested that traditional statistical methods for testing hypotheses about the importance of attributes have not been fruitful. Efforts to date have been unable to identify empirically any characteristics (image or objective), which serve to constrain competition, and thereby define submarkets. Rather, previous studies have concluded that all brands/styles compete with all other brands/styles, constrained only by their market shares. This implies that smokers without any higher order screening directly select brands/styles. Thus neither the menthol/non-menthol choice, the lifestyle image choice nor any other choice precedes the brand/style choice. But when a marketing manager portrays the product as an 'outdoors' cigarette, in a sense the manager is attempting to set the product apart from many competitors' products and thus constrain smokers' thinking to cigarettes with an 'outdoors' connotation. In other words the manager is assuming (or hoping) that some smokers favour, or can be persuaded to favour, an outdoors type of cigarette and will thus tend to constrain their choices to the set of products having this property. This, of course, would mean that a choice was made on the outdoors/indoors criterion before a brand/style is selected within the chosen 
Brand

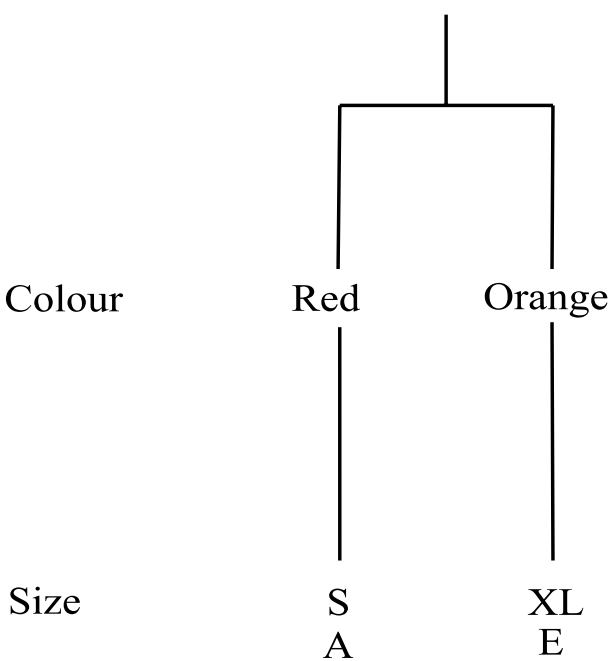

TR

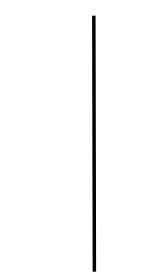

Orange

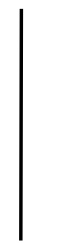

M

B
J J

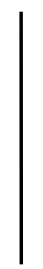

Red

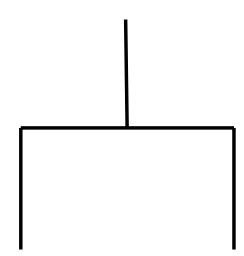

$\mathrm{S}$

$\mathrm{D}$
L

C

Figure 4 Entropy-determined attribute hierarchy

subset. In a sense, two submarkets would exist. To summarise, the empirical evidence to date has not revealed the existence of any product attributes which constitute higher order decision criteria than the rest. This runs counter to management beliefs. Therefore, the problem addressed by this study is to

Table 2: Cigarette attribute dimensions

\begin{tabular}{lll}
\hline $\begin{array}{l}\text { Attribute } \\
\text { number }\end{array}$ & Attribute dimension & $\begin{array}{l}\text { Number of } \\
\text { states }\end{array}$ \\
\hline 1 & Brand name & 37 \\
2 & Tip type & 2 \\
3 & Menthol content & 2 \\
4 & Length & 4 \\
5 & Pack type & 2 \\
6 & Taste level & 4 \\
7 & Manufacturer & 6 \\
8 & Introduction date & 4 \\
9 & Filter spin type & 7 \\
10 & Special filter type & 4 \\
11 & Tip colour & 3 \\
12 & Tar content & 7 \\
13 & Perceived tar and nicotine & 6 \\
14 & Perceived strength & 6 \\
15 & Perceived femininity & 6 \\
16 & Diameter & 2 \\
17 & Pack design & 3 \\
18 & Scene image & 3 \\
19 & Lifestyle image & 3 \\
20 & People content image & 4 \\
\hline
\end{tabular}

determine empirically whether an ordering of product attributes does exist, and if so, what that ordering is.

\section{THE CIGARETTE DATA}

The data set for the study consisted of 29,096 purchase records of cigarette products. The members of the panel kept a $\log$ of cigarette products purchased over a one-year period, recording the brand, quantity, price paid, package type, menthol content and other attributes. The string of $n$ purchased cigarette products for each respondent provided $n-1$ from-to transaction pairs. The usable data set consisted of 14,521 transaction pairs (each being a repeat purchase or switch). There were 95 brands/styles used for the study. Each cigarette product purchased was coded on each of the 20 product attributes defined in Table 2 .

\section{RESULTS}

The analysis demonstrated conclusively that there are several levels of decision making in the process by which smokers 


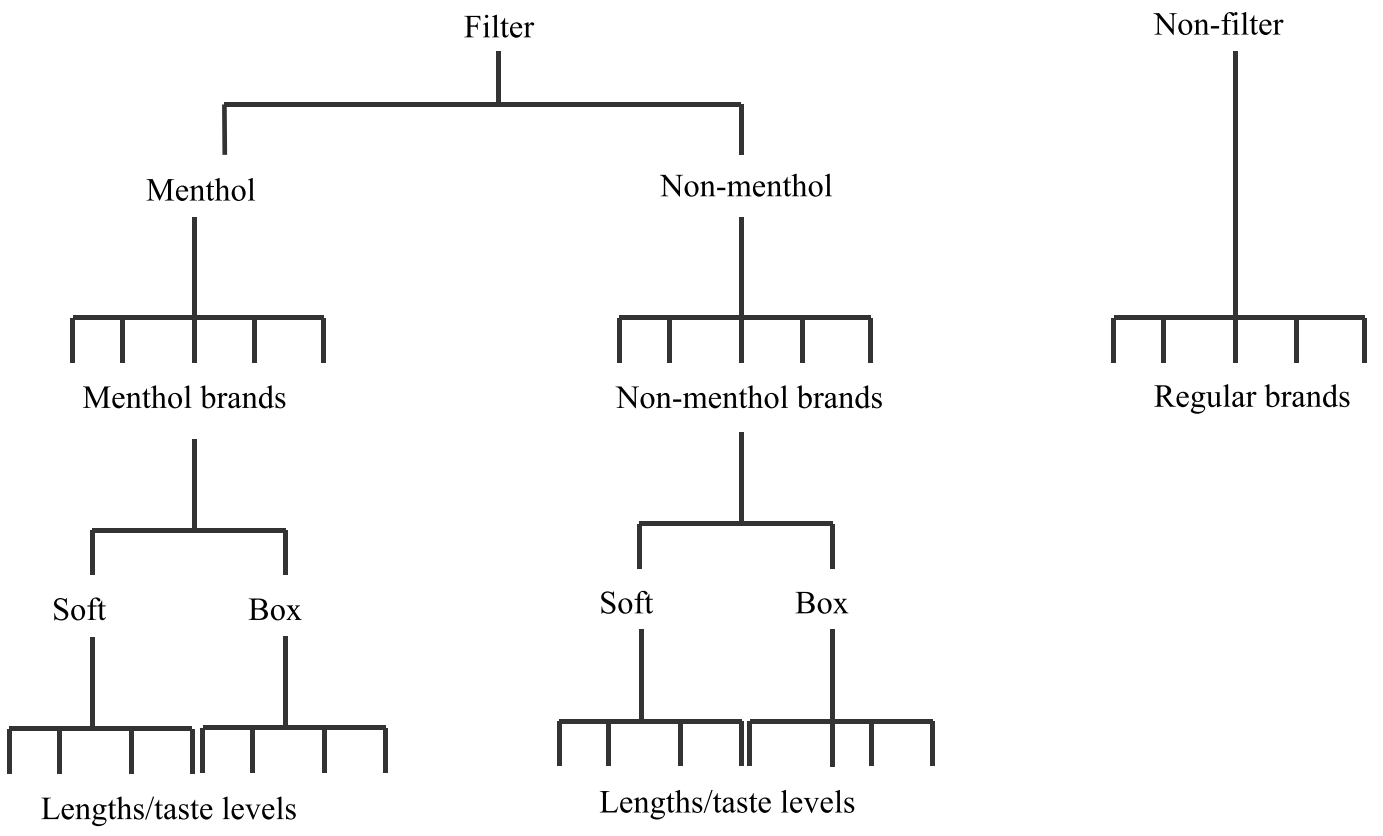

Figure 5 Hierarchical portrayal of the basic attribute structure for six objective attribute dimensions

choose cigarette brands/styles. The basic behavioural structure is depicted in Figure 5. The choice structure for the market as a whole was also found to pertain to most segments of the smoking population.

\section{Basic market structure: Objective attributes}

At the first level in the decision hierarchy is the tip type. This is clearly the most constraining influence on switching behaviour, effectively dividing the cigarette market into two submarkets. Smokers revealed a much stronger willingness to stay within their submarket (filter or non-filter) than to switch to the other.

Within the filter submarket, the second-order decision criterion is whether the product is a menthol cigarette or not. There is a significant difference between the switching entropy of the menthol content dimension and that for brand name, pack type, taste level and length.
Brand name is the third-order choice dimension. Once the brand choice is made, the switching entropy for remaining dimensions is dramatically reduced. This is to be expected in a market having a large number of brands and relatively few choices within brand, assuming of course that the tip type and menthol content choices have already been made.

Within brand name, pack type appeared as the highest-order choice dimension with taste level and length sharing the lowest place in the hierarchy.

\section{The role of image dimensions}

Particularly interesting in the analysis of the sub-brand name structure was the appearance of scene and lifestyle as greater constraints on switching than the objective dimensions: pack type, length and taste level. While only three image dimensions were analysed in the study, the middle ranking of two of them demonstrates the 
possibility that other image dimensions might well be found even higher in the structure. Should this be so, their discovery would have important implications for marketing decision making.

\section{Partitioning structure of market segments}

In addition to an overall market structure, analyses were conducted for the following market segments:

— light smokers;

- moderate smokers;

— heavy smokers;

- male;

— female;

- young (18-24);

- old $(51+)$.

One consumer segment exhibited an attribute structure that was notably different from the one for the market as a whole. Smokers in the 18-24-year age group placed a higher importance on the brand name decision. Brand name appeared at the top of the structure, followed in order by the tip type and menthol content dimensions. Ordering at the remaining levels was as for the market as a whole. The salience of brand in the attribute structure indicates greater brand loyalty relative to loyalty to other attributes for this small though strategically important segment. This finding must, however, be tempered by the fact that young smokers demonstrated a higher than average propensity to switch across all dimensions except taste level. Thus for this age group it is the relative importance of brand name that is of interest. The general market structure described all other segments, namely, light smokers, heavy smokers, male smokers and old $(51+)$ smokers.

\section{MARKETING IMPLICATIONS}

The marketing utility of the results can be suggested by comparing the structures for the overall market and the young segment. In the overall market structure, smokers think of cigarettes almost as two distinct products: filter-tipped and non-filtered. Companies with products (brands) in the two markets have to promote them separately. Furthermore, products sold under a common brand name in the two markets will not cannibalise each other. By contrast, young smokers are both more willing to experiment (they switch more than other segments) and see brands as the primary objects of their choice. They will more readily try different variants (tip or not, menthol or not) of a brand than will the rest of the market, so promotion needs to focus on encouraging young smokers to try the firm's brand, after which they will tend to remain more brand loyal (relative to other attributes) than other smokers. Promotion of the brand will affect all styles of the brand.

The implications of finding the type of scenery and lifestyle depicted in advertising below the brand choice level are less useful than if they were above brand name in the structure. Once consumers have selected the brand name, there is usually only one image associated with that brand, in which case promoting the brand and its image together is appropriate. Offering styles with different image appeals will tend to cause consumers of that brand to try these alternatives for variety, yet might increase brand share by getting some customers to switch from other brands.

\section{SUMMARY AND CONCLUSIONS}

This paper has presented a new empirical approach to market partitioning based on measuring switching entropy from switching matrices derived from diary 
panel data. The evolutionary algorithm described here contrasts with the Hendry approach and others that depend on hypothesising a partitioning structure and then comparing observed switching constants within partitions with what would be expected under equilibrium market conditions. A strength of the methodology is the ease with which the partitioning structure can be identified for any market segments of interest. Differences in partitioning structure are useful for suggesting appropriate marketing strategies for targeting different segments.

To illustrate the applicability of the methodology, results of a partitioning analysis of the cigarette market have been described. Prior to this study, discussions with industry marketing analysts had indicated that earlier studies had been unable to identify a multilevel choice structure, concluding instead that there were no higher-order choices made by smokers. The major conclusion of the present study was that a hierarchical ordering of decision criteria does exist for the cigarette market. Purchase behavioural patterns on some attribute dimensions exert significantly more constraint on switching than do others. For all segments except young smokers, the first-order decision was the choice between filter tipped and regular cigarettes. It was noted that young smokers demonstrated a greater than average switching propensity on all dimensions, but particularly between filter tipped and non-filtered product variants. This led to brand name being the first order of choice for this segment. For the market as a whole and most segments, the choice between menthol cigarettes and those without provided the second-order constraint on switching, and brand name emerged as the third-order attribute. Once the switching among brands was accounted for, there was relatively little switching among different styles of a brand. Below brand, pack type choice preceded the taste level and length choices.

Several image dimensions were included in the study. Of these, scene and lifestyle dimensions were found to exert significant constraint on post-brand name choice and in fact appeared higher in the behavioural structure than the three objective dimensions: pack type, length and taste level.

In summary, this paper has described a new method for identifying a hierarchical structure of markets for frequently-purchased products. Using data on purchase behaviour, the market can be partitioned into subsets of closely competing product variants on the basis of relative switching entropy. Knowledge of how product attributes impose constraints on consumers' choices provides the marketing manager with guidance for decisions on product positioning, promotional and pricing strategies and new product design.

\section{References}

1 Butler, D. H. and Butler, B. E. (1970, 1971) 'HendroDynamics: Fundamental laws of consumer dynamics', The Hendry Corporation.

2 The Hendry Corporation (1976) 'Speaking of Hendry', The Hendry Corporation.

3 Kalwani, M. and Morrison, D. (1977) 'A parsimonious description of the Hendry system', Management Science, Vol. 23, pp. 467-477.

4 Rubinson, J. R., Vanhonacker, W. R. and Bass, F. M. (1980) 'On "A parsimonious description of the Hendry system”, Management Science, Vol. 26, pp. 215-220.

5 Urban, G., Johnson, P. and Hauser, J. (1984) 'Testing competitive market structures', Marketing Science, Vol. 3, pp. 83-112.

6 Grover, R. and Dillon, W. (1985) 'A probabilistic model for testing hypothesized hierarchical market structures', Marketing Science, Vol. 4, pp. 312-335.

7 Kumar, A. and Sashi, C. (1989) 'Confirmatory analysis of aggregate hierarchical market structures: Inferences from brand switching behavior', Journal of Marketing Research, Vol. 26, pp. 444-453.

8 Rao, V. and Sabavala, D. (1981) 'Inference of hierarchical choice processes from panel data', Journal of Consumer Research, Vol. 8, pp. 85-96. 
9 Frank, R., Massy, W. and Wind, Y. (1973) 'Market segmentation', Prentice-Hall.

10 Winter, F. (1984) 'Market segmentation: A tactical approach', Business Horizons, January-February.

11 Bock, T. and Styles, C. (2002) 'Growing segmentation trees for strategic advantage', Business Horizons, March-April.

12 Brockett, P., Charnes, A., Cooper, W., Lerner, D. and Phillips, F. (1995) 'Information theory as a unifying statistical approach for use in marketing research', European Journal of Operational Research, Vol. 84, pp. 310-329.

13 Shannon, C. E. (1948) 'A mathematical theory of communication', Bell System Technical Journal, Vol. 27, pp. 379-423.
14 Herniter, J. (1973) 'An entropy model of brand purchase behavior', Journal of Marketing Research, Vol. 10, pp. 361-375.

15 Herniter, J. (1974) 'A comparison of the entropy model and the Hendry model', Journal of Marketing Research, Vol. 11, pp. 21-29.

16 Garner, W. and McGill, W. (1956) 'The relationship between information and variance analysis', Psychometrika, Vol. 21, pp. 219-228.

17 Brockett et al. (1995) op. cit.

$18 p_{j \mid i}=\frac{1}{n}$

$H_{\max }=-\sum_{i=1}^{n} p_{i} \sum_{j=1}^{n} \frac{1}{n} \log \frac{1}{n}=-\sum_{i=1}^{n} p_{i} \log n^{-1}=\log n$ 